KUNS-1526

$\mathrm{HE}(\mathrm{TH}) 98 / 12$

hep-lat/9808026

\title{
Axial vector current of exact chiral symmetry on the lattice
}

\author{
Yoshio Kikukawa* \\ Department of Physics, Kyoto University \\ Kyoto 606-01, Japan \\ and \\ Atsushi Yamada ${ }^{\dagger}$ \\ Department of Physics, University of Tokyo \\ Tokyo 113, Japan
}

August, 1998

\begin{abstract}
We discuss the exact chiral symmetry and its spontaneous breakdown in lattice QCD with the Dirac operators satisfying the GinspargWilson relation. The axial vector current, which turns out to be related to the vector current simply by the insertion of the operator $\gamma_{5}(1-a D)$, is explicitly constructed in the case of the Neuberger-Dirac operator. We also consider an Euclidean proof of the Nambu-Goldstone theorem using the Ward-Takahashi identity of this symmetry.
\end{abstract}

*e-mail address: kikukawa@gauge.scphys.kyoto-u.ac.jp

${ }^{\dagger}$ e-mail address: atsushi@hep-th.phys.s.u-tokyo.ac.jp 
Recently Neuberger has proposed a Dirac operator which describes exactly massless fermions on a lattice [1, 2] based on the overlap formalism of chiral determinant [3, 此. Its explicit form is known as

$$
a D_{n m}=\left(1+\gamma_{5} \frac{H}{\sqrt{H^{2}}}\right)_{n m},
$$

where $H$ is the hermitian Wilson-Dirac operator defined by

$$
H=\gamma_{5}\left\{\frac{1}{2} \gamma_{\mu}\left(\nabla_{\mu}-\nabla_{\mu}^{\dagger}\right)+\frac{a}{2} \nabla_{\mu} \nabla_{\mu}^{\dagger}-\frac{1}{a} m_{0}\right\}, \quad\left(0<m_{0}<2\right) .
$$

Here $S U(N)$ gauge group is assumed and $\nabla_{\mu}$ is the gauge covariant forward difference operator.

This Dirac operator satisfies the Ginsparg-Wilson relation [5, 6, 2, 7], 1

$$
D_{n m} \gamma_{5}+\gamma_{5} D_{n m}=\sum_{l} a D_{n l} \gamma_{5} D_{l m}
$$

This relation guarantees that the effects of the chiral symmetry breaking terms in the Dirac operator appear only in local terms and physically irrelevant. This is the clue to escape the Nielsen-Ninomiya theorem [14].

Subsequently, Lüscher pointed out that the Ginsparg-Wilson relation implies an exact symmetry of the fermion action [15]. The chiral transformation proposed is given as

$$
\delta \psi_{n}=\sum_{m} \gamma_{5}\left(1-\frac{a}{2} D\right)_{n m} \psi_{m}, \quad \delta \bar{\psi}_{n}=\sum_{m} \bar{\psi}_{m}\left(1-\frac{a}{2} D\right)_{m n} \gamma_{5}
$$

This chiral transformation can be extended to the flavor non-singlet chiral transformations. Lüscher also observed that for the flavor-singlet chiral transformation the functional integral measure is not invariant and its anomalous variant is indeed given in terms of the index of the Dirac operator.

Locality of the Neuberger-Dirac operator in the presence of gauge field is not quite obvious, although it provides a completely satisfactory local solution of the Ginsparg-Wilson relation in free theory [7, 15]. This question has been examined analytically and numerically by several authors. Hernandes, Jansen and Lüscher have given a proof of the locality for a certain set of bounded small gauge fields and also for the case with an isolated zero mode

\footnotetext{
${ }^{1}$ Another Dirac operator which satisfies the Ginsparg-Wilson relation has been proposed by Hasenfratz et. al. 6, 8, 9, 10, 11] based on the renormalization group method 12, 13. As to this fixed point Dirac operator, the authors refer the reader to the above cited references.
} 
of $H$ [16]. Locality in dynamically generated gauge fields has been examined by same authors for $S U(3)$ and by Montvay for $S U(2)$ [17]. According to the former investigation, the Neuberger-Dirac operator seems local even with the gauge fields for $\beta \geq 6.0$.

The question of the practical use of the Neuberger-Dirac operators also does not seem to be serious, although the Dirac operator involves the inverse square root of $H^{2}$ and appears at first sight to require the full storage of the size of the Dirac operator. In this respect, an approximate formula has been given by Neuberger [18], for which the conjugate gradient method for the (hermitian) Wilson-Dirac operator is applicable with the help of the shift technique for different fermion masses 19]. Other approaches have also been investigated by Chiu 20] and by Edwards, Heller and Narayanan [21].

The weak coupling perturbation theory of the massless QCD with the Neuberger-Dirac operator has been discussed by the authors [22]. The axial $U(1)$ anomaly is evaluated explicitly and is shown to have the correct form of the topological charge density for perturbative backgrounds, supplementing the original calculation in [5].

In this paper, we discuss the axial Ward-Takahashi identity associated with the exact chiral symmetry in some detail. We first consider the axial vector current which, following the Noether's procedure, is defined through the variation of the action by the "local" chiral transformation,

$$
\begin{aligned}
\delta_{5} S_{F} & =a^{4} \sum_{n m l} \bar{\psi}_{n}\left\{D_{n l} \alpha_{l} \gamma_{5}\left(1-\frac{a}{2} D\right)_{l m}+\left(1-\frac{a}{2} D\right)_{n l} \gamma_{5} \alpha_{l} D_{l m}\right\} \psi_{m} \\
& =a^{4} \sum_{n m l} \bar{\psi}_{n}\left\{D_{n l} \alpha_{l}-\alpha_{n} D_{n l}\right\} \gamma_{5}(1-a D)_{l m} \psi_{m} \\
& =a^{4} \sum_{l}\left\{\partial_{\mu} \alpha_{l} \sum_{n m} \bar{\psi}_{n} K_{l \mu}^{5}(n, m) \psi_{m}\right\}
\end{aligned}
$$

Here we have used the Ginsparg-Wilson relation to derive the second line. As we see from this structure of the variation of the action, the axial vector current may be constructed following the general procedure discussed by Ginsparg and Wilson [5], leading to the third line, where $\partial_{\mu}$ is the forward difference operator on the lattice. If In a practical point of view, however,

\footnotetext{
${ }^{2}$ Another option about the choice of the axial vector current is to use the current induced from the symmetric part of the Dirac operator $\frac{1}{2}\left[D, \gamma_{5}\right] \gamma_{5}$. In this choice, as argued by P. Hasenfratz[11], the explicit breaking term of the action do not induce the additive correction to quark mass, the renormalization of axial and vector currents and the mixing between four-fermion operators with wrong chirality, except the axial anomaly 10$]$.
} 
it would be useful to have the explicit form of the axial vector current, 3 at least, in the case with the Neuberger-Dirac operator. We will derive the explicit formula of the vector and axial vector currents in this case and also in the case with the Dirac operator in the rational approximation of Ref. [18]. Then, we discuss an Euclidean proof of the Nambu-Goldstone theorem of the spontaneous breakdown of the exact chiral symmetry, using the axial Ward-Takahashi identity.

We first note from Eq. (5) that the axial vector current has a simple relation to the vector current which is defined by

$$
\begin{aligned}
\delta S_{F} & =a^{4} \sum_{n m l} \bar{\psi}_{n}\left\{D_{n l} \alpha_{l}-\alpha_{n} D_{n l}\right\} \psi_{m} \\
& =a^{4} \sum_{l}\left\{\partial_{\mu} \alpha_{l} \sum_{n m} \bar{\psi}_{n} K_{l \mu}(n, m) \psi_{m}\right\} .
\end{aligned}
$$

Once the vector current is constructed, the kernel of the axial vector current is obtained simply by the insertion of the operator $\gamma_{5}(1-a D)$ :

$$
K_{l \mu}^{5}=K_{l \mu} \cdot \gamma_{5}(1-a D) .
$$

The above relation can naturally be understood if we recall the fact that the operator $\gamma_{5}(1-a D)$ satisfies the identity

$$
\left\{\gamma_{5}(1-a D)\right\}^{2}=1
$$

which follows from the Ginsparg-Wilson relation, as discussed by Narayanan 25] and its eigenvalues may be regarded to define another chirality analogous to $\gamma_{5}$, as considered by Hasenfratz, Niedermayer and Lüscher [26]. This chirality depends on the gauge fields. In fact, if we define the chiral components of $\psi$ and $\bar{\psi}$ through $\gamma_{5}(1-a D)$ and $\gamma_{5}$, respectively,

$$
\begin{aligned}
\gamma_{5}(1-a D) \psi_{ \pm} & = \pm \psi_{ \pm} \\
\bar{\psi}_{L, R} \gamma_{5} & = \pm \bar{\psi}_{L, R}
\end{aligned}
$$

the fermion action is decomposed into chiral parts as follows:

$$
\bar{\psi} D \psi=\bar{\psi}_{R} P_{L} D \psi_{+}+\bar{\psi}_{L} P_{R} D \psi_{-} .
$$

This leads to the factorization of the partition function into chiral determinants in the overlap formalism [25]. In the kernel of the axial vector current

\footnotetext{
${ }^{3}$ The covariant current in the overlap formalism has been discussed by Neuberger in [23], and 24. The relation between the covariant current and the Noether current obtained here will be discussed in a separate paper 27].
} 
of Eq. (7), the operator $\gamma_{5}(1-a D)$ is to assign charges to $\psi_{ \pm}$opposite each other, contrary to the vector charges.

In this respect, we also notice the following fact. For a chiral transformation of the type given by Lüscher to lead the exact symmetry of the action by the Ginsparg-Wilson relation, the weights of the Dirac operator in the transformation for $\psi$ and $\bar{\psi}$ should sum up to unity, but otherwise may be arbitrary. We can easily see by considering "local" transformations following the Noether's procedure, that all such chiral transformations lead to the same axial vector current as given by Eq. (5) and the associated axial Ward-Takahashi identities are physically equivalent. For example, the following chiral transformation also leaves the action invariant

$$
\delta \psi_{n}=\sum_{m} \gamma_{5}(1-a D)_{n m} \psi_{m}, \quad \delta \bar{\psi}_{n}=\bar{\psi}_{n} \gamma_{5}
$$

and gives more natural definition of chiral transformation in view of the chiral decomposition Eqs. (9) and (10), as discussed in [26]. We will consider this version of the exact chiral symmetry later in our discussion of the spontaneous chiral symmetry breaking.

This particular chiral symmetry, in the case of the Neuberger-Dirac operator, can be regarded as a direct consequence of the symmetry of quantum Hamiltonian in the underlying overlap formalism [3]:

$$
\begin{aligned}
\mathcal{H}_{D} & =a^{4} \sum_{n m} \hat{a}_{n}^{\dagger} H_{n m} \hat{a}_{m}-a^{4} \sum_{n m} \hat{b}_{n}^{\dagger} H_{n m} \hat{b}_{m}, \\
\mathcal{H}_{D 5} & =a^{4} \sum_{n} \hat{a}_{n}^{\dagger} \gamma_{5} \hat{a}_{n}-a^{4} \sum_{n} \hat{b}_{n}^{\dagger} \gamma_{5} \hat{b}_{n},
\end{aligned}
$$

This can be seen from the relation between the Dirac field variables $\psi_{n}$ and $\bar{\psi}_{n}$ and the grassman representatives of the canonical variables $\left(\hat{a}_{n}, \hat{a}_{n}^{\dagger}\right)$ and $\left(\hat{b}_{n}, \hat{b}_{n}^{\dagger}\right)$ [27]:

$$
\begin{aligned}
& \psi_{n}=\frac{1}{\sqrt{2}} \sum_{m}\left\{\frac{1}{2}\left(1+\frac{H}{\sqrt{H^{2}}}\right)_{n m} a_{m}+\frac{1}{2}\left(1-\frac{H}{\sqrt{H^{2}}}\right)_{n m} b_{m}\right\}, \\
& \bar{\psi}_{n}=\frac{1}{\sqrt{2}}\left\{a_{n}^{\dagger}\left(\frac{1+\gamma_{5}}{2}\right)+b_{n}^{\dagger}\left(\frac{1-\gamma_{5}}{2}\right)\right\} .
\end{aligned}
$$

Then the symmetry transformation of the canonical variables

$$
\delta \hat{a}_{n}=-\hat{a}_{n}, \quad \delta \hat{a}_{n}^{\dagger}=\hat{a}_{n}^{\dagger}, \quad \delta \hat{b}_{n}=\hat{b}_{n}, \quad \delta \hat{b}_{n}^{\dagger}=-\hat{b}_{n}^{\dagger},
$$

in fact induces Eq. (12). 
Now let us construct the vector current in the case of the NeubergerDirac operator. The covariant transformation of the Dirac operator under the local $U(1)$ transformation

$$
D_{n m} \longrightarrow D_{n m}^{\prime}=e^{-i \alpha_{n}} D_{n m} e^{i \alpha_{m}}
$$

is induced by the local transformation of the overlap Hamiltonian $H$

$$
H_{n m} \longrightarrow H_{n m}^{\prime}=e^{-i \alpha_{n}} H_{n m} e^{i \alpha_{m}}
$$

as

$$
a D_{n m}^{\prime}=\left(1+\gamma_{5} \frac{H^{\prime}}{\sqrt{\left(H^{\prime}\right)^{2}}}\right)_{n m} .
$$

Then, at the first order in the infinitesimal transformation parameter $\alpha$, we have

$$
\begin{aligned}
a D_{n m} \alpha_{m}-\alpha_{n} a D_{n m} & =\gamma_{5}\left\{\left(\frac{H}{\sqrt{H^{2}}}\right)_{n m} \alpha_{m}-\alpha_{n}\left(\frac{H}{\sqrt{H^{2}}}\right)_{n m}\right\} \\
& =\left.\gamma_{5} \delta\left(\frac{H}{\sqrt{H^{2}}}\right)_{n m}\right|_{\delta H=\left\{H_{k l} \alpha_{l}-\alpha_{k} H_{k l}\right\}},
\end{aligned}
$$

where

$$
\begin{aligned}
\delta H & =H_{n m} \alpha_{m}-\alpha_{n} H_{n m}=\sum_{l} \partial_{\mu} \alpha_{l} W_{l \mu}(n, m), \\
W_{l \mu}(n, m) & =\gamma_{5}\left\{\frac{1}{2}\left(\gamma_{\mu}-1\right) \delta_{n l} \delta_{l+\hat{\mu}, m} U_{l \mu}+\frac{1}{2}\left(\gamma_{\mu}+1\right) \delta_{n, l+\hat{\mu}} \delta_{l m} U_{l+\hat{\mu}, \mu}^{\dagger}\right\} .
\end{aligned}
$$

Using the integral representation,

$$
\frac{1}{\sqrt{H^{2}}}=\int_{-\infty}^{\infty} \frac{d t}{\pi} \frac{1}{t^{2}+H^{2}},
$$

we obtain

$$
\begin{aligned}
\delta\left(\frac{H}{\sqrt{H^{2}}}\right)_{n m} & =\delta H \frac{1}{\sqrt{H^{2}}}-H \int_{-\infty}^{\infty} \frac{d t}{\pi} \frac{1}{t^{2}+H^{2}}(H \delta H+\delta H H) \frac{1}{t^{2}+H^{2}} \\
& =\int_{-\infty}^{\infty} \frac{d t}{\pi} \frac{1}{t^{2}+H^{2}}\left(t^{2} \delta H-H \delta H H\right) \frac{1}{t^{2}+H^{2}}
\end{aligned}
$$


Therefore, the kernel of the vector current is given by

$$
a K_{l \mu}(n, m)=\gamma_{5}\left\{\int_{-\infty}^{\infty} \frac{d t}{\pi} \frac{1}{\left(t^{2}+H^{2}\right)}\left(t^{2} W_{l \mu}-H W_{l \mu} H\right) \frac{1}{\left(t^{2}+H^{2}\right)}\right\}_{n m} .
$$

It also follows from the Ginsparg-Wilson relation that the kernel of the vector current satisfies an identity given by

$$
K_{\mu} \cdot \gamma_{5}(1-a D)+(1-a D) \gamma_{5} \cdot K_{\mu}=0 .
$$

Accordingly, using the relation Eq. (7), the axial vector current is obtained as

$$
\begin{gathered}
A_{l \mu} \equiv \sum_{n m} \bar{\psi}_{n} K_{l \mu}^{5}(n, m) \psi_{m}, \\
K_{l \mu}^{5}(n, m)=\left\{K_{l \mu} \cdot\left(-\frac{H}{\sqrt{H^{2}}}\right)\right\}_{n m} .
\end{gathered}
$$

The flavor non-singlet currents can be obtained by including the generators $T^{a}$ of the rotation in the flavor space in the above expression.

Here we comment on the factorization property of the vector and axial currents into left- and right-handed currents. The vector and axial vector currents in Eqs. (5) and (6) are defined by introducing the local transformations

$$
\delta \psi_{n}=\alpha_{n} \psi_{n}, \quad \delta \bar{\psi}_{n}=-\alpha_{n} \bar{\psi}_{n}
$$

for vector current and

$$
\delta \psi_{n}=\alpha_{n} \sum_{m} \gamma_{5}(1-a D)_{n m} \psi_{m}, \quad \delta \bar{\psi}_{n}=\alpha_{n} \bar{\psi}_{n} \gamma_{5}
$$

for axial vector current, respectively, and the kernels of these currents thus constructed respect the simple relation Eq. (7). (Here we have adopted the chiral transformation Eq. (12) rather than Eq. (1) for the reason explained before.) In order to obtain the right-handed chiral current, for example, we may combine these two transformation as

$$
\delta \psi_{n}=\alpha_{n} \sum_{m}\left(P_{+}\right)_{n m} \psi_{m}, \quad \delta \bar{\psi}_{n}=-\alpha_{n} \bar{\psi}_{n} P_{L}
$$

where

$$
P_{ \pm}=\frac{1 \pm \gamma_{5}(1-a D)}{2}, \quad P_{R, L}=\frac{1 \pm \gamma_{5}}{2}
$$


Then we obtain the right-handed current as

$$
J_{l \mu}^{+}=\frac{1}{2}\left(V_{l \mu}+A_{l \mu}\right)=\sum_{n m} \bar{\psi}_{n}\left(K_{l \mu} \cdot P_{+}\right)_{n m} \psi_{m} .
$$

In the point of view of the chiral decomposition of Eqs. (9), (10) and (11), however, this current is not completely chiral since it contains not only the right-handed components $\left(\psi_{+}, \bar{\psi}_{R}\right)$ but also $\bar{\psi}_{L}$. This is because $P_{ \pm}$do not commute with the local variation operation (or operator $\delta$ ) and the above local transformation is not restricted to the right-handed chiral component $\left(\bar{\psi}_{R}, \psi_{+}\right)$only. To obtain the factorized chiral current which consists of only the right-handed components $\left(\psi_{+}, \bar{\psi}_{R}\right)$, we may consider the right-handed local chiral transformation given as follows:

$$
\delta \psi_{n}=\sum_{m l}\left(P_{+}\right)_{n l} \alpha_{l}\left(P_{+}\right)_{l m} \psi_{m}, \quad \delta \bar{\psi}_{n}=-\alpha_{n} \bar{\psi}_{n} P_{L}
$$

Here $P_{+}$in front of the local parameter $\alpha_{n}$ is necessary so that the change of the variable along the chiral transformation is consistent with the chiral decomposition:

$$
\psi_{n+}^{\prime}=\psi_{n+}+\delta \psi_{n}
$$

Then we obtain the factorized right-handed current

$$
J_{l \mu}^{R+}=\sum_{n m} \bar{\psi}_{n}\left(P_{L} \cdot K_{l \mu} \cdot P_{+}\right)_{n m} \psi_{m} .
$$

The transformation Eq. (35) gives rise to half of the covariant anomaly as the Jacobian factor of the path integral measure. The local transformation for the other component $\left(\psi_{-}, \bar{\psi}_{L}\right)$ and its current are obtained in the same way. As it should be, the axial vector like combination of these local transformations is reduced to Eq. (12) when the transformation parameter is space independent. These currents are easily generalized to the case of the flavor non-singlet chiral transformations $S U\left(N_{f}\right)_{R} \times S U\left(N_{f}\right)_{L}$.

As we can see from Eq. (15), in the case of the Neuberger-Dirac operator, the local transformation Eq. (35) can be regarded to be induced from the local transformation of the canonical variable $\left(\hat{a}_{n}, \hat{a}_{n}^{\dagger}\right)$ in the overlap formalism. Therefore the covariant and chiral (right-handed) current Eq. (37) corresponds to the covariant current in the overlap formalism [23, 24, 27].

Our expression of the axial vector current given by Eqs. (26), (28) and (29) contains an integration over the parameter $t$. For analytical studies, for example, the weak coupling expansion, this integration can be explicitly performed [22]. In numerical applications, however, we would need to treat 
it in some approximation: the integration may be replaced by a certain discrete summation. Such an approach is closely related to the rational approximation of the Dirac operator considered by Neuberger 18. In fact, if we change the variable $t$ to the variable which has a compact region as

$$
t=\tan \frac{\pi}{2} \sigma, \quad \sigma \in[-1,1],
$$

the integral representation Eq. (24), for example, may be rewritten as

$$
\frac{1}{\sqrt{H^{2}}}=\int_{-\infty}^{\infty} \frac{d t}{\pi} \frac{1}{t^{2}+H^{2}}=\int_{0}^{1} d \sigma \frac{1}{\cos ^{2} \frac{\pi}{2} \sigma} \frac{1}{\tan ^{2} \frac{\pi}{2} \sigma+H^{2}}
$$

By discretizing the variable $\sigma$ as

$$
\sigma \longrightarrow \frac{1}{n}\left(s-\frac{1}{2}\right), \quad s=1,2, \ldots, n
$$

we are lead to the rational approximation to the Neuberger-Dirac operator,

$$
a D^{(n)}=1+\gamma_{5}\left(\frac{H}{\sqrt{H^{2}}}\right)^{(n)}
$$

and

$$
\left(\frac{H}{\sqrt{H^{2}}}\right)^{(n)}=H \cdot \frac{1}{n} \sum_{s=1}^{n} \frac{1}{\cos ^{2} \frac{\pi}{2 n}\left(s-\frac{1}{2}\right)} \frac{1}{\tan ^{2} \frac{\pi}{2 n}\left(s-\frac{1}{2}\right)+H^{2}} .
$$

Similarly, we may replace the integration over the parameter $t$ in the expressions of the current kernels to the discrete summation. The same results are obtained using the expressions Eqs. (41) and (42) from the beginning. Thus we obtain the axial vector current in the case with the Neuberger-Dirac operator in the rational approximation as follows:

$$
\begin{aligned}
a K_{l \mu}^{(n)}=\gamma_{5} \frac{1}{n} \sum_{s=1}^{n} & \frac{1}{\cos ^{2} \frac{\pi}{2 n}\left(s-\frac{1}{2}\right)} \frac{1}{\tan ^{2} \frac{\pi}{2 n}\left(s-\frac{1}{2}\right)+H^{2}} \times \\
& \left(\tan ^{2} \frac{\pi}{2 n}\left(s-\frac{1}{2}\right) W_{l \mu}-H W_{l \mu} H\right) \frac{1}{\tan ^{2} \frac{\pi}{2 n}\left(s-\frac{1}{2}\right)+H^{2}} .
\end{aligned}
$$

$K_{l \mu}^{5(n)}=K_{l \mu}^{(n)}\left(-\frac{H}{\sqrt{H^{2}}}\right)^{(n)}$.

\footnotetext{
${ }^{4}$ We would like to thank H. Neuberger for suggesting this relation.
} 
It may also be possible to use the expansion in Legendre polynomials for the inverse square root of $H^{2}$ as adopted by Hernandes, Jansen and Lüscher [28, 16]. With the help of the integral representation Eq. (39), we can derive the expansion of the current kernels in terms of Legendre polynomials.

Finally, we consider the axial Ward-Takahashi identity associated with the flavor non-singlet chiral transformation Eq. (12),

$$
\delta \psi_{n}=\sum_{m} T^{a} \gamma_{5}(1-a D)_{n m} \psi_{m}, \quad \delta \bar{\psi}_{n}=\bar{\psi}_{n} \gamma_{5} T^{a} .
$$

In particular, we discuss the chiral multiplet of the scalar and pseudo-scalar density operators considered by Neuberger, Hasenfratz and Chandrasekharan [7, 11, 29],

$$
\delta\left(\bar{\psi}_{m} \gamma_{5} \psi_{m}\right)=2 \bar{\psi}_{m} \sum_{l}\left(1-\frac{a}{2} D\right)_{m l} \psi_{l} .
$$

Its axial Ward-Takahashi identity is given as follows:

$$
a^{4} \partial_{\mu}^{*}\left\langle A_{n \mu}^{a} \bar{\psi}_{m} T^{b} \gamma_{5} \psi_{m}\right\rangle+\delta_{n m} \delta^{a b}\left\langle\bar{\psi}_{m} \sum_{l}\left(1-\frac{a}{2} D\right)_{m l} \psi_{l}\right\rangle=0 .
$$

Here we assume that the vacuum expectation values are defined by the functional integrals over both the fermion and the gauge fields. In the case of the Neuberger-Dirac operator, we also assume that the gauge fields satisfies the smoothness condition discussed in [16] by the choice of an appropriate gauge field action. Note that, with this constraint, the kernel of the axial vector current can be shown to be exponentially (uniformly) bounded [16].

Then the identity implies that the massless pole would appear in the correlation function of the axial vector current and the pseudo scalar density $\left(\hat{p}_{\mu} \equiv \frac{2}{a} \sin \frac{a p_{\mu}}{2}\right)$

$$
\text { F.T. }\left\langle A_{n \mu}^{a} \bar{\psi}_{m} T^{b} \gamma_{5} \psi_{m}\right\rangle(p)=-i \frac{\hat{p}_{\mu}}{\hat{p}^{2}} \delta^{a b}\left\langle\bar{\psi}_{m} \sum_{l}\left(1-\frac{a}{2} D\right)_{m l} \psi_{l}\right\rangle,
$$

if the spontaneous breakdown of the exact chiral symmetry occurs

$$
\left\langle\bar{\psi}_{m} \sum_{l}\left(1-\frac{a}{2} D\right)_{m l} \psi_{l}\right\rangle \neq 0
$$


This consists an Euclidean proof of the Nambu-Goldstone theorem on the lattice. Let us assume the coupling of the Nambu-Goldstone boson to the axial vector current and the pseudo scalar density as follows:

$$
\begin{aligned}
\left\langle 0\left|A_{n \mu}^{a}\right| \pi^{b}(p)\right\rangle & =\delta^{a b} F_{\pi} i \hat{p}_{\mu} e^{i p n}, \\
\left\langle 0\left|\bar{\psi}_{n} T^{a} \gamma_{5} \psi_{n}\right| \pi^{b}(p)\right\rangle & =\delta^{a b} a^{-2} Z_{\pi}^{\frac{1}{2}} e^{i p n} .
\end{aligned}
$$

Then we obtain the relation,

$$
F_{\pi} Z_{\pi}^{\frac{1}{2}}=-a^{2}\left\langle\bar{\psi}_{m} \sum_{l}\left(1-\frac{a}{2} D\right)_{m l} \psi_{l}\right\rangle
$$

which is well-known in the continuum theory.

Comments about the choice of the multiplet of the scalar and pseudo scalar density operators are in order. Under the "global" chiral transformation Eq. (12), it is easily verified that the following scalar and pseudo scalar density operators consist an exact chiral multiplet [26]:

$$
S_{n}=\bar{\psi}_{n} \sum_{m}\left(1-\frac{a}{2} D\right)_{n m} \psi_{m}, \quad P_{n}=\bar{\psi}_{n} \sum_{m} \gamma_{5}\left(1-\frac{a}{2} D\right)_{n m} \psi_{m},
$$

as

$$
\delta S_{n}=2 P_{n}, \quad \delta P_{n}=2 S_{n} .
$$

Then one might think of the axial Ward-Takahashi identity which involves the pseudo scalar density $P_{n}$. The above chiral property, however, is not reflected directly in the axial Ward-Takahashi identity, because it is derived through the "local" chiral transformation. In fact, what we obtain in this case is as follows:

$a^{4} \partial_{\mu}^{*}\left\langle A_{n \mu} P_{m}\right\rangle+\delta_{n m}\left\langle S_{m}\right\rangle+\left\langle\bar{\psi}_{m} \gamma_{5}\left(1-\frac{a}{2} D\right)_{m n} \sum_{l} \gamma_{5}\left(1-\frac{a}{2} D\right)_{n l} \psi_{l}\right\rangle=0$.

The third term in the l.h.s. is not proportional to $\delta_{n m}$ times the chiral condensate, contrary to Eq. (47). As far as the axial Ward-Takahashi identity for the multiplet of the scalar and pseudo scalar densities is concern, the choice of Chandrasekharan gives rise to the simple identity.

In summary, we have considered the exact chiral symmetry and its spontaneous breakdown in lattice QCD with the Dirac operators satisfying the Ginsparg-Wilson relation. We have constructed explicitly the axial vector current in the cases with the Neuberger-Dirac operator and its rational 
approximation, which turns out to be related to the vector current simply by the insertion of the operator $\gamma_{5}(1-a D)$. Then we have considered an Euclidean proof of the Nambu-Goldstone theorem with the axial WardTakahashi identity associated with the "local" chiral transformation. The relation given by Eq. (52) or the explicit formula of the axial vector current may be useful in the numerical evaluation of the pion decay constant $F_{\pi}$.

\section{Acknowledgments}

We are indebted to H. Neuberger for enlightening discussions. Y.K. would like to thank M. Lüscher for the communication concerning the locality of Neuberger's Dirac operator. Y.K. is supported in part by Grant-inAid for Scientific Research from Ministry of Education, Science and Culture(\#10740116,\#10140214). A.Y. would like to thank Pamela Morehouse for sending him an useful preprint.

\section{References}

[1] H. Neuberger, Phys. Lett. B417 (1998) 141.

[2] H. Neuberger, Phys. Lett. B427 (1998) 353.

[3] R. Narayanan and H. Neuberger, Nucl. Phys. B412 (1994) 574; Phys. Rev. Lett. 71 (1993) 3251; Nucl. Phys. B443 (1995) 305.

[4] R. Narayanan and J. Nishimura, Nucl. Phys. B508 (1997) 371; Y. Kikukawa and H. Neuberger, Nucl. Phys. B513 (1998) 735.

[5] P. H. Ginsparg and K. G. Wilson, Phys. Rev. D25 (1982) 2649.

[6] P. Hasenfratz, Nucl. Phys. B(Proc. Suppl.) 63 (98) 53.

[7] H. Neuberger, Phys. Rev. D57 (1998) 5417.

[8] W. Bietenholz, R. Brower, S. Chandrasekharan, U.-J. Wiese, Nucl. Phys. Proc. Suppl. 53 (1997) 921.

[9] T. DeGrand, A. Hasenfratz, P. Hasenfratz, P. Kunszt and F. Niedermayer, Nucl. Phys. Proc. Suppl. 53 (1997) 942.

[10] P. Hasenfrats, V. Laliena and F. Niedermayer, The index theorem in QCD with a finite cut-off, hep-lat/9801021. 
[11] P. Hasenfratz, "Lattice QCD without tuning and mixing and current renormalization", hep-lat/9802007.

[12] P. Hasenfrats and F. Niedermayer, Nucl. Phys. B414 (1994) 78; T. DeGrand, A. Hasenfratz, P. Hasenfratz and F. Niedermayer, Nucl. Phys. B454 (1995) 587.

[13] U.-J. Wiese, Phys. Lett. B315 (1993) 417; W. Bietenholz and U.J. Wiese, Nucl. Phys. B(Proc. Suppl.) 34 (1994) 516.

[14] N.B. Neilsen and M. Ninomiya, PL B105 (1981) 219; Nucl. Phys. B185 (1981) 20 [E: B195 (1982) 541]; ibid B193 (1981) 173.

cf. Friedan, Commun. Math. Phys. 85 (1982) 481.

[15] M. Lüscher, Phys. Lett. B428 (1998) 342.

[16] P. Hernandes, K. Jansen and M. Lüscher, hep-lat/9808010.

[17] R. Kirchner, S. Luckmann, I. Montvay, K. Spanderen, J. Westphalen, "Numerical Simulation of Dynamical Gluinos: Experience with the Multi-Bosonic Algorithm", poster given at the International Symposium on Lattice Field Theory, Boulder, July 13-18, 1998.

[18] H. Neuberger, "A practical implementation of the Overlap-Dirac operator", hep-lat/9806025.

[19] A. Frommer, S. Güsken, T. Lippert, B. Nöckel, K. Schilling, Int. J. Mod. Phys. C6 (1995) 627.

[20] T.-W. Chiu, "Topological Charge and The Spectrum of Exactly Massless Fermions on the Lattice", hep-lat/9804016.

[21] R.G. Edwards, U.M. Heller and R. Narayanan, "A Study of Practical Implementations of the Overlap-Dirac Operator in Four Dimensions", hep-lat/9807017.

[22] Y. Kikukawa and A. Yamada, "Weak coupling expansion of massless QCD with a Ginsparg-Wilson fermion and axial U(1) anomaly", hep-lat/9806013. For the weak coupling expansion in the overlap in general, see A. Yamada, Phys. Rev. D57 (1998) 1433, Nucl. Phys. B514 (1998) 399, hep-lat/9802013, to appear in Nucl. Phys. B.

[23] H. Neuberger, "Geometrical aspects of chiral anomalies in the overalp", hep-lat/9802033. 
[24] H. Neuberger, "Lattice chirality", talk given at the International Symposium on Lattice Field Theory, Boulder, July 13-18, 1998. hep-lat/9807009.

[25] . R. Narayanan, "Ginsparg-Wilson relation and the overlap formula" , hep-lat/9802018.

[26] F. Niedermayer, P. Hasenfratz and M. Lüscher, plenary talk given by F. Niedermayer at the International Symposium on Lattice Field Theory, Boulder, July 13-18, 1998.

[27] Y. Kikukawa and A. Yamada, "An exact lattice chiral symmetry in the overlap formalism", in preparation.

[28] B. Bunk, Nucl. Phys. B(Proc. Suppl.) 63 A-C (1998) 952.

[29] S. Chandrasekharan, "Lattice QCD with Ginsparg-Wilson fermions", hep-lat/9805015. 